\title{
Experimental Protocol for Assessing the Relation Between Indoor Air Quality and Living Unit Conditions in AAL
}

\author{
Milica VUJOVIĆ*, Milan RISTANOVIĆ, Marko MILOŠ
}

\begin{abstract}
The presented study intends to demonstrate experimental protocol of indoor air quality measurements in diverse living environments, how they relate with the existing living conditions and affect future design of ambient assisted living systems. By addressing this specific issue, this study tends to reinforce the awareness about the issues related to the indoor air quality in the ambient assisted living field of research. The proposed experimental protocol includes an overview of the aspects, such as space size, ventilation, heating cleaning and immediate outdoor air quality, that need to be measured in the living environment in order to gain insight into the changes of indoor air quality through a comprehensive information dataset. The proposed experimental protocol describes the procedures for preparing the experiment, as well as collecting and analyzing data. Mentioned independent variables related to the living environment conditions and dependent variables that include temperature, humidity, carbon dioxide $\left(\mathrm{CO}_{2}\right.$ ), volatile organic chemicals (VOCs), PM2.5 (atmospheric particulate matter smaller than 2.5 microns) and PM10 (atmospheric particulate matter smaller than 10 microns) are defined, as is statistical analysis that should examine interdependencies.
\end{abstract}

Keywords: ambient assisted living; experimental setting; indoor air quality

\section{INTRODUCTION}

Ambient Assisted Living (AAL) is a very broad subarea of Intelligent Buildings. It uses information and communication technologies in the automation of certain processes within the residential environments with the aim to provide assistance to the users in their everyday life. This specific category covers different types of users, but primarily refers to people who spend more time indoors, and therefore for this study, the population of elderly people presents the target group [1]. The aim is to enable users to remain healthy and comfortable for as long as possible through environmental and behavioral monitoring systems, providing the necessary care and detecting early signs of certain life-threatening conditions or situations. This technology includes systems integrated into the environment as well as mobile systems that users carry with them [2]. The amount and complexity of user requirements in this field is considerable and a commitment to creating efficient and reliable systems is essential. It is also important to note that developments in this field over the past decade have been significant [3], where user awareness of these systems has been enhanced. Users no longer see such systems as help related to their incapability of taking care of themselves, but as a reflection of the general awareness and benefits of using technology to improve quality of life [4].

An important part of AAL field are the systems for measuring indoor air quality (IAQ) [5] which have recently become even more relevant. Their main role is reflected in the timely and accurate measurement of data [6] that will inform other AAL systems such as Heating, Ventilation and Air Conditioning (HVAC) systems, security systems and notification systems (i.e. for healthcare actions). Many studies indicate the influence of IAQ on various health and cognitive parameters in humans $[7,8]$. As the elderly are a specific category where particular attention should be paid to these aspects of life, the importance of monitoring the IAQ status is increased [9]. Respiratory problems are one of the first consequences of poor air conditions, and due to reduced movement of the target group and longer stay in the indoor environment [10], it is evident that this problem requires special attention. Also, studies show that the air quality that people breathe is directly related to their physical [11] and mental states [12].

Research into indoor air quality has begun as a consequence of increasing interest into outdoor air quality and the results that these studies have produced [13]. Research has suggested that indoor air is much more polluted than outdoor air [14]. The differences that occurred in differently urbanized (inhabited) settlements led to the conclusion that the quality of the air in the buildings, as well as in their immediate surroundings, needs to be examined more closely in order to discover the causes behind the possible differences [15]. Further studies have shown that indoor air quality plays a large role in the quality of life [16] as well as in causing certain undesirable conditions and diseases [17, 18], which motivated this study to create an experimental design that would allow the collection of adequate data to inform design practice in the AAL domain. However, challenges in IAQ research exist and they are reflected in new kinds of pollutants (both in outdoor and indoor environments) and different lifestyles [24]. Further challenges of IAQ measurements with elderly are related to their cognitive states and the issues that may arise from cognitive disabilities [20]. Equipment issues present another challenge of the IAQ research [25]. High quality equipment will reduce the possibility of noise appearance and disconnection of devices, which presents a relevant asset when equipment cannot be monitored throughout the whole experiment. Contribution to the already vast field of research would be in obtaining and structuring information related to connection between IAQ parameters and living environment conditions of elderly people.

Experimental design is a powerful methodology for establishing cause-and effect relationships between variables [25]. Study explains [26] how experimental design uses statistical analysis to determine relationships between explanatory (independent) variables and outcome (dependent) variables. Therefore, experimental design presents an approach suitable for understanding the relationship between IAQ and living conditions of the 
elderly, which is why it is used in creating experimental setup presented in this study.

This study focuses on investigating the differences in indoor air quality in different housing units where elderly people live. As they are users who spend more time indoors, the impact of indoor air quality on their physical and mental health is greater. Recent research shows possible correlations between various aspects of living conditions, such as cleaning methods [19], ventilation systems [20] and type of heating [21] to be influential on the quality of the air inside living environments. Therefore, it is clear that the long-term exposure to faulty cleaning, ventilation or heating habits could lead to the serious degradation of user's health. Investigation of these occurrences has been present in the AAL research field [22], and provided substantial proof that there are certain dependencies, but further research has to be comprehensive in order to provide clearer answers about the causes of problems. Furthermore, besides examining these issues with the objective of removing potential hazards from living environments, there is a necessity for durable guidelines in terms of AAL systems design.

The aim of this study is to analyze the dependence between indoor air quality and different indoor conditions and contribute to the expansion of knowledge in the field of AAL by discussing potential implications that should be considered. This paper presents the first part of the study, where detailed experimental design has been created. Health aspects of users will be recorded only informatively, but not analyzed in more detail within this study. Environmental factors such as outdoor air quality in the immediate environment, the type of ventilation system, the total floor area, the total window area, the type of heating system and the type of cleaning products will be treated as the independent variables in the design of the experiment. Parameters of outdoor air quality that will be measured are Carbon Monoxide (CO), Nitrogen Dioxide $\mathrm{NO}_{2}$, Ozone $\mathrm{O}_{3} 1$ - hour avg, Ozone $\mathrm{O}_{3} 8$ - hour avg, $\mathrm{PM}$ 10 (atmospheric particulate matter than 10 microns), PM 2.5 (atmospheric particulate matter smaller than 2.5 microns) and Sulfur Dioxide $\mathrm{SO}_{2}$. They will also be treated as independent variables and will be related to each considered living environment. It is important to mention that VOCs are numerous and this study focuses on the ones containing carbon-containing organic chemicals present in indoor air, which come from a large number of indoor sources including building materials, furnishings, consumer products, tobacco smoking, people and their activities, and indoor chemical reactions.

These environmental factors have shown to have significant influence on the indoor air quality. Dependent variables, which are expected to show differences in indoor air quality between independent variables, will include temperature, humidity, carbon dioxide $\left(\mathrm{CO}_{2}\right)$, volatile organic chemicals (VOCs), PM2.5 (atmospheric particulate matter smaller than 2.5 microns) and PM10 (atmospheric particulate matter smaller than 10 microns) as parameters of indoor air quality. By analyzing mentioned dependencies, we will address the following research question:

- $\quad$ RQ: What type of experimental setting is needed to comprehensively obtain and structure the information related to indoor air quality with the aim to improve the AAL design?

This study aims to answer the research question by presenting the concept of experimental design that integrates qualitative and quantitative analysis of indoor air quality data. The study relates the indoor air quality data to the characteristics of the living environment and generates novel insight into this aspect of AAL research. The experimental design concept presented here contributes by providing a structured data acquisition methodology that would generate a comprehensive dataset for informed decision making in the design process of AAL systems in assisted living environments.

\section{METHOD}

\subsection{Overview}

Proposed experimental design (Fig. 1) is based on the findings and conclusions from previous research, as listed above. Indoor air quality greatly contributes to the various aspects of quality of life and should be included as an important factor in the design and operation of the AAL systems. The measurements of indoor air quality parameters and the factors affecting it are a complex process and the introduction of this process into the design flow and operation of AAL systems must be well structured. The proposed experimental design should test the setup, where the experimental output parameters should have an adequate format and a wealth of information. Proposed experimental design is tested with open IAQ dataset [23] and simulated values for living environment characteristics. The presented values are informative and they do not provide realistic measurements taken. Their main role in this paper is to demonstrate the process of examining the relations between living environment conditions and indoor air quality. They should demonstrate how to include data into analysis and comprehensively analyze all parameters. Following paragraphs provide a detailed explanation on how data would be gathered in a real setting and the results present the main concept of data analysis with the mentioned data taken from the open source.

\subsection{Participants}

Participants in the experiment would be persons over 65 years of age, from different living situations (living alone, living in a couple, living with family) and from different living environment conditions. Each housing unit considered in this study provides only one participant in the experiment. Participation in the experiment is voluntary. Participants with cognitive disabilities where qualitative data cannot be collected (and there is no caretaker to provide it) will not be included in the experiment.

\subsection{Measurements and Materials}

All measurements are carried out in the living quarters of the experiment participants. Experimental design involves the use of sensors, questionnaires, interviews and observation as a means of gathering information. Used materials are part of the experimental design developed in 
this study and they are supported by previously mentioned literature.

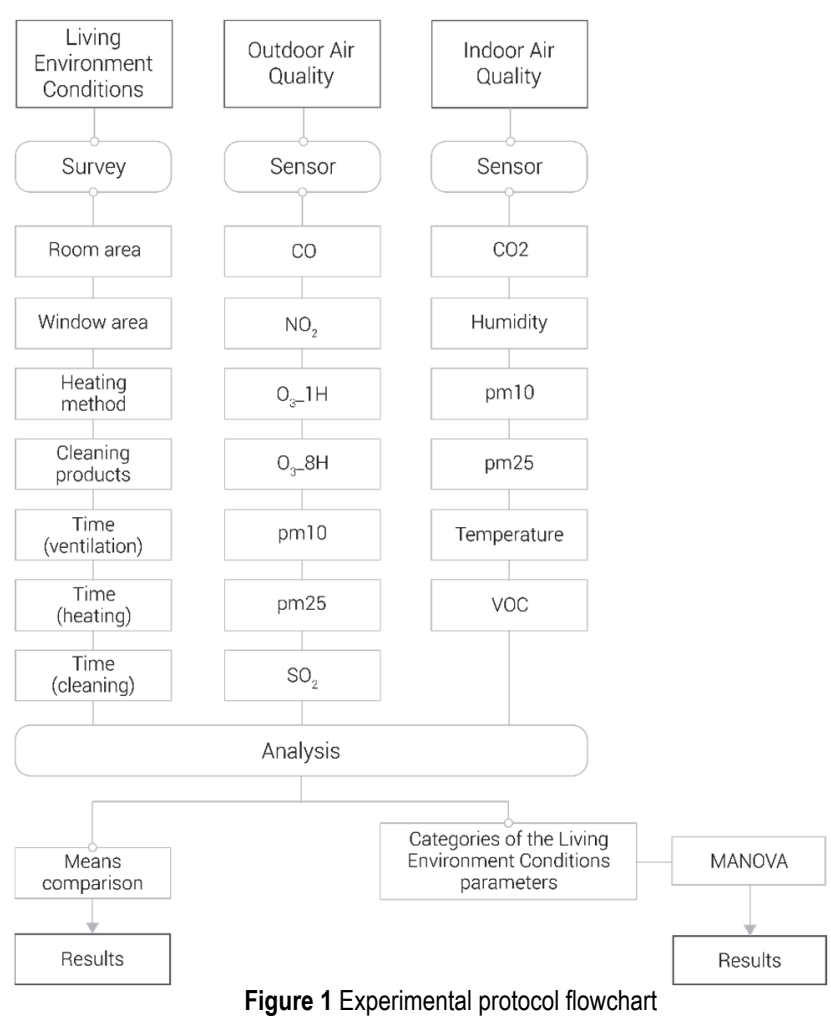

The IAQ sensor is placed in the room where the participant spends the most of his/her time during the day (If there are more rooms, the measurement is repeated for each of them. Time is not divided by the number of rooms). The measurement takes 7 days. Observations and surveys are taken right before and just after the experiment where independent variables are collected. Tab. 1 shows how each of the variables is measured, where the independent variables are combined to be measured by a sensor for measuring indoor air quality. Independent variables were obtained through questionnaires, interviews and observation. Questions related to the types of heating and ventilation, as well as questions about the areas of floor and windows are open-ended so that the participant or the interviewer can enter the information. In cases where participants cannot provide information on the area, researchers will conduct the measurement. Two options (entering or measuring the values) are left for participants to decide, as her/his own decision is related to privacy concerns. Questions related to the frequency and duration of actions are structured in a way that the collected data can be quantified and later compared with quantitative measurements. This means that the time unit for expressing the frequency of ventilation will be "per day" and frequency of cleaning and heating will be "per week".

Table 1 Independent and dependent variables

\begin{tabular}{|l|l|}
\hline Independent variables & \\
\hline Outdoor air quality in the immediate environment & \\
\hline Type of ventilation system (window, duct) & \\
\hline Type of heating system (electrical, central, wood) \\
\hline Total room area & \multicolumn{2}{|c|}{ Outdoor air quality sensors } \\
\hline Total window area & \\
\hline Type of cleaning products & \\
\hline Frequency of ventilation & \\
\hline Duration of ventilation per occurrence & \\
\hline Frequency of cleaning & \\
\hline Duration of cleaning per occurrence & \\
\hline Frequency of heating & \\
\hline Duration of heating per occurrence & \\
\hline Dependent variables & \\
\hline Temperature & \\
\hline Humidity & \\
\hline Carbon dioxide $\left(\mathrm{CO}_{2}\right.$ ) & \\
\hline Volatile organic chemicals (VOCs) & \\
\hline PM2.5 (atmospheric particulate matter smaller than 2.5 microns) \\
\hline
\end{tabular}

Table 2 Survey that collects the information on the living conditions

\begin{tabular}{|c|l|}
\hline \multicolumn{2}{|l|}{ Usage and characteristics of living environment-survey } \\
\hline 1. & What is the area of the room you spend most of your day? \\
\hline 2. & What is the total area of the windows in your apartment? \\
\hline 3. & What is the area of the windows in the room where you spend most of your day? \\
\hline 4. & $\begin{array}{l}\text { Which type of ventilation do you use in your house (opening the windows, non-automatic ventilation ducts, automatic ventilation ducts etc.)? } \\
\text { Please write your answer and elaborate if necessary. }\end{array}$ \\
\hline 5. & $\begin{array}{l}\text { Which type of heating system is used in your house (electrical heating, central heating etc.)? Please write your answer and elaborate if } \\
\text { necessary. }\end{array}$ \\
\hline 6. & Which cleaning product do you use in your house? Please write your answer and elaborate if necessary. \\
\hline 7. & For how long you ventilate the space during the day in winter months (if the frequency is lower, it can be expressed in "times per week")? \\
\hline 8. & For how long do you heat the space during the day in winter months (if the frequency is lower, it can be expressed in "times per week")? \\
\hline 9. & For how long do you clean the space during the day in winter months (if the frequency is lower, it can be expressed in "times per week")? \\
\hline
\end{tabular}

Tab. 2 shows the survey that would collect the information on the living conditions for each participant of the experiment and provide dependent variable data. Answers to the question will be post-processed in a way to obtain string or numerical values suitable for the statistical analysis software. Therefore, the required areas will be numerical values. Types of heating system, ventilation and cleaning products will be one-word instances. Duration of ventilating, heating and cleaning will be expressed in time units per day, except in cases where the action is less 
frequent when it can be expressed in units of time per week. All qualitative data that cannot be expressed in numerical values will be quantified either by Likert scale application or by categorization of the answers and assigning category numbers to each answer. Due to the limited size of the paper, detailed data processing protocol is excluded. As a contingency plan for data loss, several actions are considered. Both Data measured with sensors and qualitative data will be checked immediately after the recording and backed up at the same time. If some data is missing, the recording will be repeated, and time assigned for that will be included in the experiment session.

\subsection{Analysis}

The quantitative and qualitative data collected during the experiment will be analyzed using two analysis methods-mean comparison and multivariate analysis of variance (MANOVA). Air quantitative data, comparison of mean values for each dependent variable should indicate which living environment condition produces better indoor air quality parameters. This simple analysis should only imply clues for the deeper examination of the causes of pollution. Multivariate analysis of variance (MANOVA) considers several dependent variables by testing the difference in two or more vectors of means which provides deeper insight compared to means comparison. In other words, we are testing the hypotheses regarding the effect of one or more independent variables on two or more dependent variables. Null hypothesis here would be to claim that indoor air quality parameters (expressed through dependent variables) are not affected by living conditions (independent variables) and we will see if the null hypothesis will be rejected. Results from qualitative analysis will later be triangulated with results from quantitative analysis in order to provide deeper insight into the obtained findings.

Table 3 Presentation of prospective results with the aim of explaining the experimental protocol

\begin{tabular}{|c|c|c|c|c|c|c|}
\hline \multicolumn{7}{|c|}{ Living environment conditions } \\
\hline subject & 1 & 2 & 3 & 4 & 5 & 6 \\
\hline room area & 10 & 12 & 11 & 22 & 25 & 30 \\
\hline window area & 2 & 3 & 1.3 & 3 & 2 & 1.3 \\
\hline heating method & electricity & central & central & electricity & wood & central \\
\hline cleaning product & $\mathrm{A}$ & $\mathrm{B}$ & $\mathrm{A}$ & $\mathrm{A}$ & $\mathrm{A}$ & $\mathrm{B}$ \\
\hline time (ventilation) & 3 & 5 & 1 & 2 & 1 & 3 \\
\hline time (heating) & 10 & 8 & 7 & 8 & 10 & 8 \\
\hline time (cleaning) & 1 & 0.5 & 1 & 2 & 1 & 0.5 \\
\hline \multicolumn{7}{|c|}{ Outdoor air quality } \\
\hline $\mathrm{CO}$ & 0.62 & 0.55 & 0.48 & 0.70 & 0.62 & 0.58 \\
\hline $\mathrm{NO}_{2}$ & 42.68 & 29.73 & 22.00 & 28.42 & 31.43 & 23.69 \\
\hline $\mathrm{O}_{3} \_1 \mathrm{H}$ & 119.56 & 102.86 & 38.96 & 73.58 & 76.64 & 83.31 \\
\hline $\mathrm{O}_{3}-8 \mathrm{H}$ & 119.32 & 110.36 & 41.83 & 69.67 & 73.14 & 83.31 \\
\hline pm10 & 56.09 & 35.65 & 11.26 & 38.92 & 47.86 & 42.15 \\
\hline pm25 & 47.50 & 26.18 & 7.17 & 23.83 & 21.50 & 23.31 \\
\hline $\mathrm{SO}_{2}$ & 11.04 & 9.27 & 8.00 & 10.46 & 12.00 & 9.54 \\
\hline \multicolumn{7}{|c|}{ Indoor air quality } \\
\hline $\mathrm{CO}_{2}$ & 1184.88 & 1099.06 & 1162.87 & 659.86 & 1200.67 & 872.44 \\
\hline humidity & 58.13 & 43.36 & 42.15 & 41.40 & 41.10 & 39.36 \\
\hline pm10 & 6.29 & 6.77 & 5.19 & 32.58 & 6.08 & 19.52 \\
\hline pm25 & 5.77 & 6.26 & 4.79 & 30.75 & 5.30 & 18.03 \\
\hline temperature & 25.66 & 22.60 & 24.61 & 20.72 & 24.95 & 25.11 \\
\hline VOCs & 0.07 & 0.06 & 0.07 & 0.09 & 0.08 & 0.12 \\
\hline
\end{tabular}

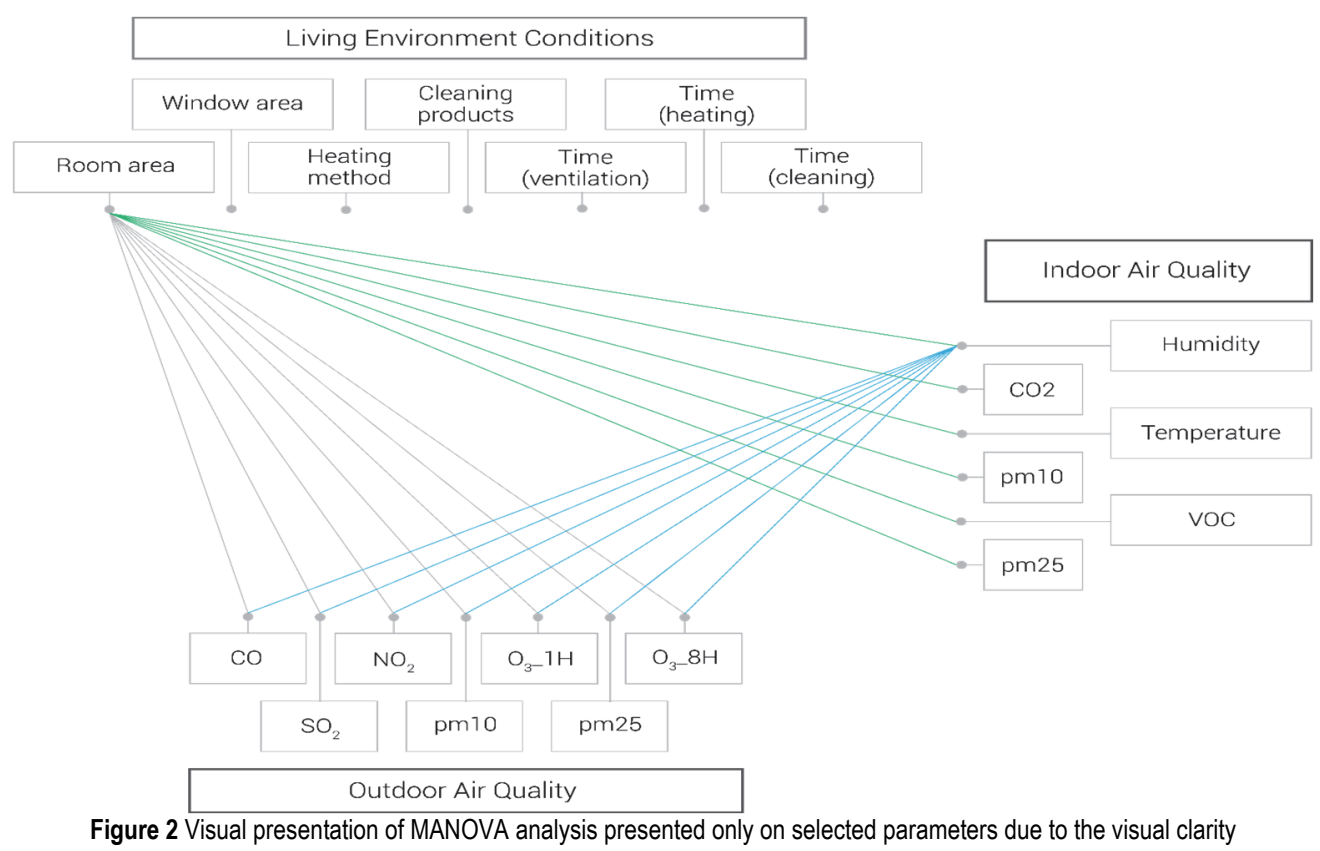




\section{RESULTS FROM THE SIMULATION}

Presented data (Tab. 3) only simulates the results with the aim to illustrate the procedure, where the IAQ data displays differences between conditions of living environment and outdoor air quality. Mean values can indicate certain dependencies and provide general conclusions about the influences of various living conditions. In the presented example, IAQ for two living environments displayed higher levels of unwanted elements, which could imply that the combination of conditions could cause the pollution. Together with outdoor air quality, the information can be triangulated and input for the AAL system could be generated. Namely, findings from the obtained analysis can be used for creating the AAL system working protocol. Definition of this protocol is not within the scope of this paper. However, this paper proposes steps if more detailed analysis could be required, depending on the needs of the future studies in AAL systems. Therefore, the MANOVA analysis is adopted, that provides more detailed insight and Fig. 2 illustrates the conceptual application of it.

\section{DISCUSSION AND LIMITATIONS}

Presented paper addresses the research question: What type of experimental setting is needed to comprehensively obtain and structure the information related to indoor air quality with the aim to improve the AAL design? The experimental protocol elaborated here introduces a structured approach to comprehensively detecting the relationship between certain indoor air quality parameters and indoor environmental factors for the specific context (living space of elderly people). The presented method is modular, which means it can be expanded with additional variables, depending on the need for the output of the individual research case. However, this study purposefully focuses on the limited variety of data aiming at creating feasible protocol that can later be expanded. The experimental protocol also aims to provide data that would address the insufficient correlation between the actual state of the living environments and the AAL systems used and overcome this issue by optimizing the operation of AAL systems. This paper does not discuss the specifics of AAL benefits that could be obtained from the results of this experimental protocol, due to the focus on the experimental protocol itself. Specific benefits will be the subject of the future work. Therefore, the paper focuses on the comprehensiveness of the methodology to obtain and structure the available information on the indoor air quality.

The methodology has certain limitations. Because of the specific characteristics of the target group, it is difficult to obtain high number of participants. Also, it should be taken into account that there are additional parameters that can influence but are difficult to measure. It is difficult to group categories of users so that anyone can be treated as a case study rather than a user study, which allows validity to be conducted with a small number of participants.

\section{CONCLUSION AND FUTURE WORK}

Proposed experimental protocol provides grounding for conducting analysis of IAQ that should inform AAL systems. It presents the first step in the research study that collects various aspects in one place and addresses dependencies between living environment, outdoor air quality and indoor air quality at the same time.

Future work considers conducting the experiments in a real setting by applying presented experimental protocol and testing real data in the statistical analysis process. In other words, a certain number of subjects will be included in the experiment and the parameters in their living space will be measured. In this way, the experimental protocol will be tested. Iterations are expected to optimize the protocol. The limited number of data types presented in this paper will potentially be augmented and adapted to each new context where the experimental protocol is applied. However, any complication of the experiment should be feasible, due to the established robust methodology.Findings should provide initial guidelines for the design practice of AAL.

\section{REFERENCES}

[1] Almeida-Silva, M.,Wolterbeek, H., \& Almeida, S. (2014). Elderly exposure to indoor air pollutants. Atmospheric Environment, 85, 54-63.

https://doi.org/10.1016/j.atmosenv.2013.11.061

[2] Bhattacharya, S., Sridevi, S., \& Pitchiah, R. (2012). Indoor air quality monitoring using wireless sensor network. 2012 Sixth International Conference on Sensing Technology (ICST). https://doi.org/10.1109//CSensT.2012.6461713

[3] GANESAN, B., GOWDA, T., AL-JUMAILY, A., FONG, K., MEENA, S., \& TONG, R. (2019). Ambient assisted living technologies for older adults with cognitive and physical impairments: a review. European Review for Medical and Pharmacological Sciences, 23, 10470-10481.

[4] Blackman, S., Matlo, C., Bobrovitskiy, C., Waldoch, A., Fang, M. L., Jackson, P., \& Sixsmith, A. (2016). Ambient Assisted Living Technologies for Aging Well: A Scoping Review. Journal of Intelligent Systems, 25(1), 55-69. https://doi.org/10.1515/jisys-2014-0136

[5] Preethichandra, D. (2013). Design of a smart indoor air quality monitoring wireless sensor network for assisted living. 2013 IEEE International Instrumentation and Measurement Technology Conference (I2MTC). https://doi.org/10.1109//2MTC.2013.6555624

[6] Pitarma, R., Marques, G., \& Ferreira, B. R. (2016). Monitoring indoor air quality for enhanced occupational health. Journal of Medical Systems, 41(2). https://doi.org/10.1007/s10916-016-0667-2

[7] WHO European Centre for Environment and Health. Report on the WHO Technical Meeting On Quantifying Disease From Inadequate Housing. Bonn, Germany: WHO European Centre for Environment and Health; 2006.

[8] Minnesota. Department of Public Service, American Society of Heating, Refrigerating, Air-Conditioning Engineers, \& American National Standards Institute. (1988). Air leakage performance for detached single-family residential buildings, 119(1988). American Society of Heating, Refrigerating and Air-conditioning Engineers.

[9] Shin, H., Park, W., Kim, B., Ji, K., \& Kim, K. T. (2018). Indoor Air Quality and Human Health Risk Assessment for Un-regulated Small-sized Sensitive Population Facilities. Journal of Environmental Health Sciences, 44(4), 397-407. 
[10] Pramitasari, D. \& Sarwadi, A. (2019). Vulnerability Of Elderly In Their Home Environment At High Densely Settlement. Nature: National Academic Journal of Architecture, 6(1), 1. https://doi.org/10.24252/nature.v6i1a1

[11] Wolkoff, P. (2018). Indoor air humidity, air quality, and health-An overview. International journal of hygiene and environmental health, 221(3), 376-390. https://doi.org/10.1136/oemed-2018-ICOHabstracts.550

[12] Zhang, X., Wargocki, P., Lian, Z., \& Thyregod, C. (2016). Effects of exposure to carbon dioxide and bioeffluents on perceived air quality, self-assessed acute health symptoms, and cognitive performance. Indoor Air, 27(1), 47-64. https://doi.org/10.1111/ina.12284

[13] Pegas, P. N., Evtyugina, M. G., Alves, C. A., Nunes, T., Cerqueira, M., Franchi, M., \& Freitas, M. D. (2010). Outdoor/indoor air quality in primary schools in Lisbon: A preliminary study. Química Nova, 33(5), 1145-1149. https://doi.org/10.1590/S0100-40422010000500027

[14] Sundell, J. (2004). On the history of indoor air quality and health. Indoor Air, 14(S7), 51-58. https://doi.org/10.1111/j.1600-0668.2004.00273.x

[15] Cincinelli, A. \& Martellini, T. (2017). Indoor Air Quality and Health. International Journal of Environmental Research and Public Health, 14(11). https://doi.org/10.3390/ijerph14111286

[16] Wyon, D. P. (2004). The effects of indoor air quality on performance and productivity. Indoor Air, 14, 92-101. https://doi.org/10.1111/j.1600-0668.2004.00278.x

[17] Tuomainen, A., Seuri, M., \&Sieppi, A. (2004). Indoor air quality and health problems associated with damp floor coverings. International Archives of Occupational and Environmental Health, 77(3), 222-226. https://doi.org/10.1007/s00420-003-0481-2

[18] Mendes, A., Bonassi, S., Aguiar, L., Pereira, C., Neves, P., Silva, S., \& Teixeira, J. P. (2015). Indoor air quality and thermal comfort in elderly care centers. Urban Climate, 14, 486-501. https://doi.org/10.1016/j.uclim.2014.07.005

[19] Wei, W., Boumier, J., Wyart, G., Ramalho, O., \& Mandin, C. (2015). Cleaning practices and cleaning products in nurseries and schools: To what extent can they impact indoor air quality? Indoor Air, 26(4), 517-525. https://doi.org/10.1111/ina.12236

[20] Bentayeb, M., Norback, D., Bednarek, M., Bernard, A., Cai, G., Cerrai, S., \& Annesi-Maesano, I. (2015). Indoor air QUALITY, ventilation and respiratory health in elderly residents living in nursing homes in Europe. European Respiratory Journal, 45(5), 1228-1238. https://doi.org/10.1183/09031936.00082414

[21] Annesi-Maesano, I., Baiz, N., Banerjee, S., Rudnai, P., Rive, S., \& The Sinphonie Group. (2013). Indoor air quality and sources in schools and related health effects. Journal of Toxicology and Environmental Health, Part B, 16(8), 491550. https://doi.org/10.1080/10937404.2013.853609

[22] Almeida-Silva, M., Wolterbeek, H., \& Almeida, S. (2014). Elderly exposure to indoor air pollutants. Atmospheric Environment, 85, 54-63. https://doi.org/10.1016/j.atmosenv.2013.11.061

[23] GAMS Indoor Air Quality Dataset. (2019). Available online: https://github.com/twairball/gams-dataset.

[24] Tham, K. W. (2016). Indoor air quality and its effects On humans-A review of challenges and developments in the last 30 years. Energy and Buildings, 130, 637-650. https://doi.org/10.1016/j.enbuild.2016.08.071

[25] Fraenkel, J. R., Wallen, N. E., \& Hyun, H. H. (1993). How to design and evaluate research in education (Vol. 7). New York: McGraw-hill.

[26] Brown, S. R., \& Melamed, L. E. (2007). Experimental design and analysis. Newbury Park, CA: Sage Publ.

\section{Contact information:}

Milica VUJOVIC, M.Sc.ME, M.Arch.

(Corresponding author)

University of Belgrade, Faculty of Mechanical Engineering,

KraljiceMarije 16, Belgrade, Serbia

E-mail: milicavujovic.mv@gmail.com

Milan RISTANVIC, Dipl-Ing. Professor

University of Belgrade, Faculty of Mechanical Engineering, KraljiceMarije 16, Belgrade, Serbia

E-mail: mristanovic@mas.bg.ac.rs

Marko MILOS, Dipl-Ing. Professor

University of Belgrade, Faculty of Mechanical Engineering, KraljiceMarije 16, Belgrade, Serbia

E-mail: mmilos@mas.bg.ac.rs 